\title{
The Effectiveness and Tolerability of Oral Acetaminophen/Aspirin/Caffeine (AAC) Combination Regimen as an Acute Treatment for Migraine in Adults: A Meta-Analysis of Randomized Trials
}

\author{
Adrian I. Espiritu, ${ }^{1}$ Huckie C. Del Mundo, ${ }^{2}$ Jonas Daniel P. Bico ${ }^{2}$ and Paul Matthew D. Pasco ${ }^{1}$ \\ ${ }^{1}$ Department of Neurosciences, College of Medicine and Philippine General Hospital, University of the Philippines Manila \\ ${ }^{2}$ College of Medicine, University of the Philippines Manila
}

\begin{abstract}
Background. Migraine is a highly common disorder that can cause significant disability on an individual, which collectively may lead to a substantial burden for the society. Various expert societies have recommended Acetaminophen/Aspirin/Caffeine (AAC) combination regimen as the first-line drug treatment for migraine attacks; however, there were no pooled evidences summarizing the effectiveness and tolerability of this regimen.
\end{abstract}

Objective. To determine the effectiveness and tolerability assessment of oral AAC combination regimen as an acute treatment for migraine in adults.

Methods. Relevant studies from inception to March 2014 were searched in Cochrane CENTRAL, MEDLINE, LILACS, Scopus and metaRegister of Controlled Trials. The Cochrane Collaboration's tool for the assessment of risk of bias was employed. Trials that were randomized, double-blind, parallel-group, placebo and active-controlled were included and the data were employed for meta-analysis. To evaluate the quality of evidence, the GRADE approach was utilized for outcomes with sufficient studies and data.

Results. From 225 records identified, 4 trials were included in this review, with a total of 3,608 participants with recorded baseline characteristics. Patient-reported migraine intensity was moderate-severe and the AAC dose used was at 500/500/130 mg. At 2 hours, AAC regimen was statistically different and found to be superior to placebo in terms of pain-free, headache relief, nausea-free, photophobia-free, phonophobia-free and functional disability reduction rates using intension-to-treat analysis. Missing data did not alter the outcome measures generating robust results. Sumatriptan $100 \mathrm{mg}$ was found to be better than AAC in pain-free rate, and phonophobia-free rates at 2 hours. Statistically more patients in the AAC arm experienced "any adverse event" compared to placebo and complaints were commonly nausea and nervousness.

Presented at the 13th Interns' Research Forum, April 22, 2014, UP College of Medicine, University of the Philippines Manila.

Corresponding author: Adrian I. Espiritu, MD

Department of Neurosciences

Philippine General Hospital

University of the Philippines Manila

Taft Ave. Ermita, Manila, 1000 Philippines

Telephone: +639258520341

Email: espirituadrian@gmail.com
Conclusion. For adult individuals with moderate-severe migraine, a fixed oral dose of Acetaminophen/Aspirin/Caffeine (AAC 500/500/130 mg) may be used as first-line therapy for the acute treatment of migraine and is only associated with mild, infrequent adverse events.

Key Words: Acetaminophen/Aspirin/Caffeine, migraine without aura, migraine with aura, migraine disorders

\section{Introduction}

Migraine is a paroxysmal primary headache disorder frequently associated with nausea, light and sound hypersensitivities, and/or other less common features such as vomiting and odor sensitivity. The overall prevalence rate of individuals with migraine including other persons with severe headaches among Americans ranges approximately from $16 \%$ to $23 \% .^{1}$ It is more common among females ages $18-44$, in whites more than blacks, and those with lower household income..$^{1-3}$ Nearly $50 \%$ of persons experiencing severe headaches can trigger considerable amount of impairment in activities or can lead to bed rest. ${ }^{2}$

The therapeutic agents used in the acute treatment of migraine belong to a wide array of drug classes, which mainly include the simple analgesics (non-steroidal antiinflammatory drugs (NSAIDs) and acetaminophen), the triptans, the antiemetics, and the ergots. From this pool of anti-migraine drugs, the effects of acetaminophen, aspirin and caffeine for the abortive therapy of migraine were considered in this review.

Acetaminophen, with its analgesic effects, although it has insignificant anti-inflammatory activity, may block nociceptive information in the thalamus and may modify the prostaglandin, substance $\mathrm{P}$, serotonin, and arginine nitric oxide synthetase systems. ${ }^{4}$ Combined with the similar action of aspirin on COX-2, the beneficial analgesic effect is ostensibly intensified further. ${ }^{4}$ Lastly, caffeine has been used with other simple analgesics including acetaminophen and aspirin, and other migraine-specific agents, such as ergotamine, due to its beneficial adjuvant properties. ${ }^{5} \mathrm{~A}$ rational consequence of these positive effects is to combine acetaminophen, aspirin and caffeine into a fixed regimen for the acute treatment of migraine.

Currently, there are no extensive systematic reviews or meta-analyses summarizing the effectiveness and tolerability 
of acetaminophen/aspirin/caffeine regimen as a symptomatic treatment for migraine in adults with particular regard to the risk of bias or the methodological quality of the relevant trials. Hence, this particular review may provide the best evidence-based report on this particular intervention by employing the rigorous guidelines indicated in the Cochrane Handbook for Systematic Reviews of Interventions and in the PRISMA (Preferred Reporting Items for Systematic reviews and Meta-analyses) Statement. ${ }^{6-7}$

\section{Methods}

\section{Criteria for the selection of studies}

Trials reported as primary research, expressed in the English language, and available in full-text articles were considered in this review. Studies that employed randomized, at least double-blind, parallel group, placeboand/or active-controlled designs were included. Trials which involved adults (at least 18 years of age) with a diagnosis of migraine (with or without aura) that generally conforms with the International Classification of Headache Disorders (ICHD) criteria were incorporated in this review. ${ }^{8}$ No restrictions were implemented in terms of migraine severity or intensity, frequency and duration, or use of any prophylactic therapy for migraine. Studies that utilized the Acetaminophen/Aspirin/Caffeine (AAC) combination regimen (as the intervention of interest) taken as a single combined or multiple oral regimens given for the acute (abortive or symptomatic) treatment of migraine were considered in this review. There were no restrictions set on this intervention in terms of dosage and timing of administration (e.g. during prodromal, aura or headache phase, or generally early in the attack or after the attack has fully developed).

Studies utilizing other designs, such as quasiexperimental (non-randomized controlled design and pre/post-test design), cluster-randomized, cross-over, prospective/retrospective cohort, case-control and crosssectional trials, were excluded. Trials which reported secondary data (i.e. data extracted from other trials) and in which no full-text report is accessible were excluded. Any duplicate reports of the same study, abstract-only reports, results with no accompanying background and methods, reviews, and animal studies were excluded. Trials employing "no treatment" as comparison were also excluded. Studies that were intended to determine the prophylactic effectiveness of the intervention in reducing migraine frequency were excluded.

\section{Primary and secondary outcomes}

To determine the most significant and most consistent outcome measure terms across trials, outcomes were selected based on the investigator's guidelines of the International Headache Society on the controlled trials of drugs in migraine. ${ }^{9}$

\section{Search methods for the identification of studies}

From inception to March 2014, the following databases were searched for relevant articles: the Cochrane Central Register for Controlled Trials (CENTRAL) by The Cochrane Library, MEDLINE by PubMed, Scopus, LILACS (Literatura Latino-Americana e do Caribe em Ciências da Saúde), and metaRegister of Controlled Trials (mRCT). The general search and corresponding MeSH terms which were used include the following: ((acetaminophen OR paracetamol) AND (aspirin OR "acetylsalicyclic acid" OR acetylsalicylate) AND (caffeine OR coffee)) AND ("migraine with aura" OR "migraine without aura" OR migrain* OR headach* OR cephalgi* OR cephalalgi* OR nause* OR vomit* OR photophobia OR phonophobia) AND (randomized control* trial* OR control* clinical trial* OR clinical trial ${ }^{*}$ OR random $^{*}$ OR placebo* OR trial* OR groups OR assign* OR allocat* OR volunteer*). Other available databases, including The HERDIN Database of the Philippines, websites of the Philippine Neurological Association (PNA) and the Philippine College of Physicians (PCP), were searched. Reference lists of relevant articles (retrieved studies and review articles) were explored for other possible significant trials.

\section{Data collection and analysis}

Formulated screening and eligibility criteria as well as data extraction forms were initially pilot-tested prior to utilization. Two review authors (AIE, HCDM) independently screened the titles and abstracts of all possible relevant trials using the screening criteria. All relevant trials were then retrieved in full-text articles and were reviewed by two authors (AIE, JDPB) using the eligibility criteria. Two authors (JDPB, HCDM) independently collected the data from the included studies.

Risk of bias assessment was independently performed by two review authors (AIE, HCDM). The Cochrane Collaboration's tool for the assessment of risk of bias was employed for this review.

Risk ratios (RR) of benefit or harm with $95 \%$ confidence intervals were used to compare measures of treatment effect for all dichotomous outcomes. The "number needed to treat for an additional beneficial outcome" (NNTB) or the "number needed to treat for an additional harmful outcome" (NNTH) with 95\% confidence intervals were also computed depending on the effects of the study treatments. The unit of analysis was the individual patient.

For missing dichotomous data, imputation was performed by assuming that either the missing participants did experience the event or the missing participants did not experience the event in the intervention or control groups, yielding "best-case" and "worst-case" scenarios, respectively.

Clinical heterogeneity was assessed by considering the populations, interventions, comparisons and outcome measures in all the included studies. Methodological heterogeneity was evaluated by comparing the study designs and the risk of bias in the trials. Statistical 
heterogeneity was detected using the chi-squared $\left(X^{2}\right)$ test with a p-value $<0.10$ to indicate statistically significant heterogeneity. The degree of heterogeneity was measured using $\mathrm{I}^{2}$ statistic with values 0 to $40 \%$ indicating unimportant statistical heterogeneity. ${ }^{6}$ Constructing a funnel plot was not done since the number of included studies is less than 10 .

Two review authors (AIE, HCDM) analyzed independently the collected data using Review Manager (RevMan) 5.2.10 Outcome measures were studied using perprotocol (PPA) and/or intention-to-treat (ITT) analyses depending on the available data. Based on the information in the published articles, the review authors undertook reinclusion and re-exclusion of missing data if deemed reasonable, generating a modified ITT population. Dichotomous outcomes were combined using the MantelHaenzel method. ${ }^{6}$ A meta-analysis was performed using fixed-effect model. ${ }^{6}$ A statistical significant difference between the intervention and the control was noted if the $95 \% \mathrm{CI}$ of the relative risks of benefit or harm did not include the number one.

Sensitivity analysis on the effects of missing data was performed.

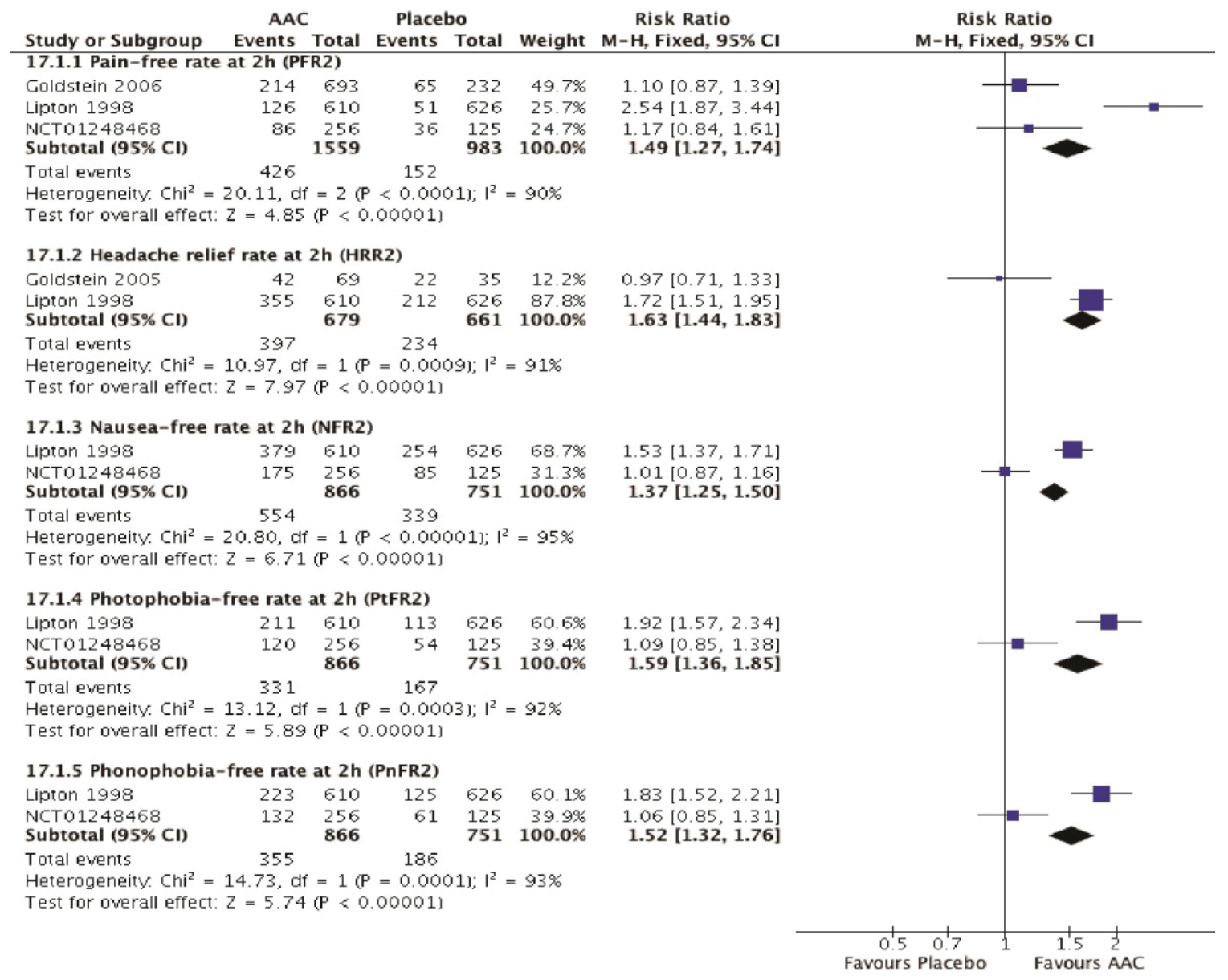

Figure 2. Forest plots of comparison AAC versus placebo for the primary outcome "Pain-free rate at 2h" (PFR2), and for the secondary outcomes, which include "Headache relief rate at 2h" (HRR2), "Nausea-free rate at 2h" (NFR2), "Photophobia-free rate at $2 \mathrm{~h}$ " (PtFR2) and "Phonophobia-free rate at 2h" (PnFR2).
In order to assess the confidence to which one can believe the published evidences on the effect estimate of the intervention, the GRADE (Grades of Recommendation, Assessment, Development and Evaluation) approach was employed for outcomes with sufficient studies and data. ${ }^{6}$ Using this approach, a Summary of Findings (SoF) table was constructed which presents key findings in this review in a self-explanatory and transparent format.

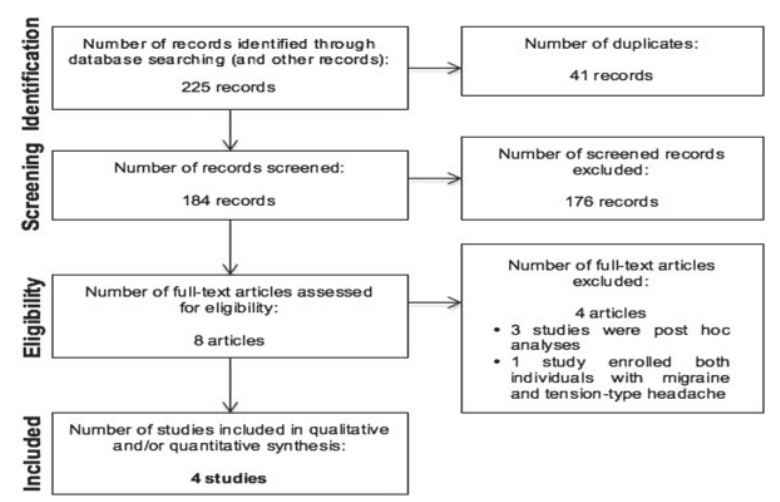

Figure 1. Flow diagram of information of the review. 


\section{Results}

There were 5 databases that were explored extensively for potential relevant articles. Using the particular search strategies for the various databases, the search generated a total of 225 records (CENTRAL: 27 records; PubMed: 66; Scopus: 125; mRCT: 7; LILACS: 0). No relevant trials were obtained in the HERDIN, and websites of the PNA and PCP. Figure 1 shows the schematic diagram of information flow in this review.

There were 4 studies that fulfilled the inclusion criteria established for this review. Three studies were published in peer-reviewed journals and one study (identified as NCT01248468) was accessed using metaRegister of Controlled Trials (mRCT) database in the clinicaltrials.gov website. ${ }^{11-14}$

Forest plots comparing AAC and placebo in various outcome measures are shown in Figure 2. Sensitivity analyses on the effect of missing data were investigated (data not shown). The Summary of Findings table for the important outcomes in the comparison of AAC and placebo is shown in Table 1.
For the primary outcome of this review, pain-free rate at 2h (PFR2), AAC regimen was found to be better than placebo, with an NNTB of 9 (3 studies; 2542 participants; RR $1.49\left[95 \%\right.$ CI 1.27, 1.74]). ${ }^{12-14}$ However, AAC was not statistically different from Ibuprofen $400 \mathrm{mg}$ (1 study; 1391 participants; RR 0.93 [95\% CI 0.80, 1.08]). ${ }^{12}$ Sumatriptan 100 $\mathrm{mg}$ dose was found to be better than the AAC regimen (1 study; 517 participants; RR 0.73 [95\% CI 0.59, 0.91]). ${ }^{14}$ For headache relief rate at $2 \mathrm{~h}$ (HRR2), AAC was superior to placebo, with an NNTB of 5 (2 studies; 1340 participants; RR 1.63 [95\% CI 1.44, 1.83]). ${ }^{11,13}$ No statistical difference was noted for AAC compared to Ibuprofen 400 mg (1 study; 1391 participants; RR 1.01 [95\% CI 0.94, 1.10]) and to Sumatriptan 50 mg (1 study; 136 participants; RR 0.99 [95\% CI 0.76, 1.30]). ${ }^{11,12}$ In the reduction of migraine-associated symptoms, which includes nausea, photophobia, and phonophobia at 2 hours, AAC was better than placebo with an NNTB of 6 (RR 1.37 [95\% CI 1.25, 1.50]), 7 (RR 1.59 [95\% CI 1.36, 1.85]), and 7 (RR 1.52 [95\% CI 1.32, 1.76]), respectively, based on 2 studies with 1617 participants. ${ }^{13,14}$ When AAC regimen was compared to Sumatriptan $100 \mathrm{mg}$, statistically more

Table 1. Summary of findings (SoF) table for the main comparison

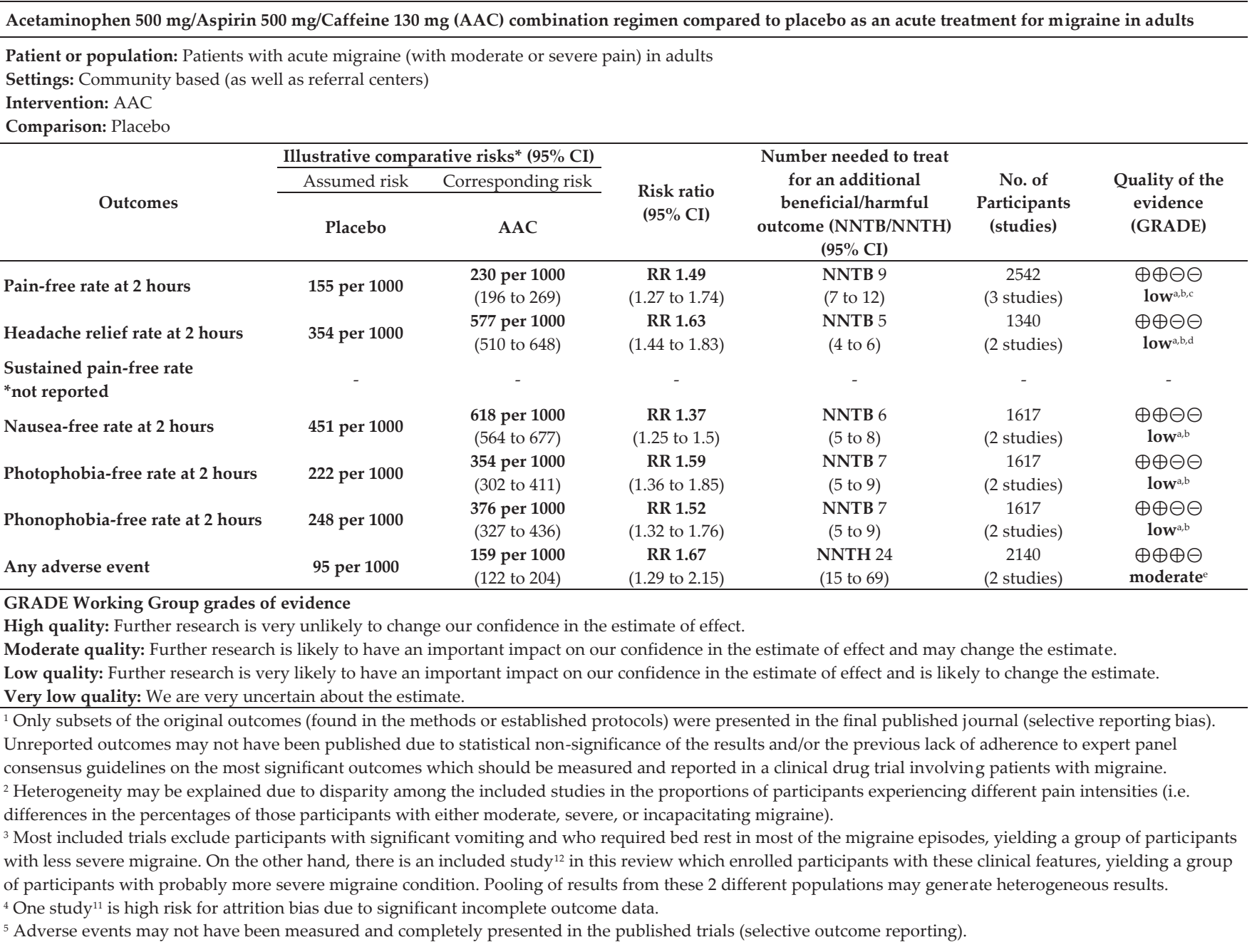


participants experienced phonophobia-free rate at 2 hours in the latter than the former regimen ( 1 study; 517 participants; RR 0.84 [95\% CI 0.72, 0.97]). ${ }^{14}$ There were very limited data to compare the reduction of migraine-associated symptoms in comparisons AAC versus Ibuprofen $400 \mathrm{mg}$ and AAC versus Sumatriptan $100 \mathrm{mg}$. In terms of decreasing functional disability at 2 hours, AAC was better than placebo with an NNTB of 5 (1 study; 1236 participants; RR 1.67 [95\% CI 1.47, 1.90]). ${ }^{13}$ Interestingly, AAC was observed to have higher relapse rate (RR) than to either placebo (1 study; 104 participants; RR 6.09 [95\% 1.53, 24.29]) or Sumatriptan $50 \mathrm{mg}$ (1 study; 136 participants; RR 7.77 [95\% CI 2.45, 24.59]). ${ }^{11}$ However, it should be noted that the data were only obtained from a very small sample size (i.e. wide 95\% CI) and the trial from which the data were collected suffers greatly from high incomplete outcome data with a computed attrition rate at $34.6 \%$. Thus, these data should be interpreted with caution.

Adverse events (AEs) were commonly described as mild and generally low in incidence. When the interventions were compared with the control groups, very few particular AEs yielded significant results. No serious AEs were noted in all the included studies. Statistically more patients in the AAC experienced "any adverse event" than placebo (2 studies; 2140 participants; NNTH 24; RR 1.67 [95\% CI 1.29, $2.15]){ }^{12,13}$ The greatest proportion of these events may be gastrointestinal-related (2 studies; 994 participants; NNTH 36; RR 2.48 [95\% CI 1.08, 5.72]). ${ }^{11,12}$ Among the specific AEs, nausea (3 studies; NNTH 63; RR 2.33 [95\% CI 1.34, 4.06]) and nervousness (2 studies; 2140 participants; NNTH 42; RR 5.24 [95\% CI 2.21, 12.38]) generated significant results. ${ }^{12-14}$ Curiously, more patients in the placebo arm experienced vomiting than in the AAC regimen arm (2 studies; 2140 participants; NNTH 103; RR 0.22 [95\% CI 0.06, 0.86]). ${ }^{12,13}$ It may be hypothesized that this phenomenon might be associated with the untreated migraine symptom complex in the placebo arm (as well as in the case of the symptom nausea).

\section{Discussion}

This review included 4 significant trials which were generated from the systematic search and evaluation of 225 records from 5 major journal and citation index databases (Figure 1). All the included studies employed randomized, double-blind, parallel-group methodological design (Table 1). The intervention given was consistent across included studies, which is a single oral dose of 2 tablets of Acetaminophen/Aspirin/Caffeine or AAC at $250 \mathrm{mg}, 250 \mathrm{mg}$, and $65 \mathrm{mg}$, respectively, yielding a total dose of $500 \mathrm{mg}, 500$ $\mathrm{mg}$, and $130 \mathrm{mg}$ for each agent. Aside from the placebo control, the intervention was also compared head-on with 2 other accepted anti-migraine drugs, which included Ibuprofen at $400 \mathrm{mg}$ dose and Sumatriptan at doses $50 \mathrm{mg}$ and $100 \mathrm{mg}$. The study population (total sample $=3608$ ) was mostly female $(81 \%)$, mean age range at 36.7 to 38.3 years, originating from the white race $(81 \%)$, with migraine without aura as the most prevalent migraine type (80\%). The usual pain intensity and usual disability associated with migraine were moderate $(27 \%)$ to severe $(72 \%)$. In addition, the population was greatly represented by patients taking nonprescription or over-the-counter anti-migraine drugs $(53 \%)$.

All trials employed the IHS criteria for migraine diagnosis. Patients were recruited by both population-based (e.g. random-digit dialing) and conventional methods (private practice patients, referrals, local advertising). Performance of these recruitment methods allowed enlisting of extensive range of patients with migraine including those individuals who do not seek medical care. ${ }^{12}$

Three trials excluded those participants with more severe migraine (i.e. those requiring bed rest for more than $50 \%$ of migraine episodes). ${ }^{11,13,14}$ In addition, those patients presenting with vomiting $20 \%$ of the time were excluded because of the reduced probability of absorbing the given intervention via the oral route. These measures may have decreased the representativeness of the participants belonging to the upper spectrum of migraine severity (i.e. patients with more severe debilitating migraine). At the lower end of the spectrum, patients with mild severity of migraine were not enrolled in the trials (i.e. the migraine pain intensity must be at least of moderate intensity). Therefore, these inclusion and exclusion criteria may have reduced the applicability of the results of this review for these underrepresented populations.

All included trials used a process of randomization to balance the baseline characteristics in their treatment arms; however, two trials were not able to indicate the particular method of random sequence procedure.12,14 In terms of allocation sequence concealment, all trials were not able to indicate the concealment method used to protect the allocation sequence before and until assignment. Although, there was no indication in all reports that this was performed, the generated baseline characteristics of each study revealed that participants in both the intervention of interest and the control treatment were similar. This provides ancillary support that all the studies were likely low risk for selection bias. Nevertheless, the review authors decided to assign "unclear risk" in the "allocation sequence concealment" for each trial for the lack of direct evidence in the published reports.

All studies employed double-blinding (i.e. patient, investigator); however, its meaning is slightly controversial when stated only in a clinical trial without proper definition because it may mean masking the patients and the clinician (the one who gave the study medications) and probably no effort was done to mask the outcome assessors (the one who collects and verifies the outcomes reported by the participants). In the case of the trials included in this review, 
the review authors presumed that the "investigator" includes both the clinician and the outcome assessor and thus, these trials were assigned "low risk" for performance and detection biases.

Attrition rate for most studies was generally low ranging from 1.3 to $6.1 \%$, except for 1 study which had a significantly high attrition rate at $34.6 \% .{ }^{11}$ The denominator used to calculate for the attrition rate was derived from the "modified intention-to-treat (ITT) population". Common reasons for attrition, in which participants were re-included in the ITT sample, comprised of "lost to follow-up", "no baseline" and "no postbaseline" evaluations. "Headache not migraine" patients were commonly not excluded in the ITT sample and thus re-excluded. One study was not able to sufficiently present the causes of its high missing data in the published report. ${ }^{11}$

For the selective reporting bias, 3 trials did not present the full raw data results in the published reports even though the outcomes had been specified in the methods or protocol. ${ }^{11-13}$ Only one trial presented all the primary and secondary outcomes in the report as stated in the protocol yielding a low risk for reporting bias for this study. ${ }^{14}$ All included trials reported "any adverse event" associated with the study intervention.

It is noteworthy that some placebo rates in the outcome measures were relatively high. This may be explained by the small sample size of the placebo as a result of high randomization ratio. In this randomization scheme, the participants have a greater chance of being allocated to the active treatment than placebo and consequently, the participants are more probable to believe receiving the active intervention. ${ }^{12}$ This strategy is usually done for ethical reasons but at the risk of increased placebo response. ${ }^{16,17}$

When AAC was compared to placebo across studies, statistical heterogeneity existed on the results of the important outcomes, which included pain-free, headache relief, nausea-free, photophobia-free, and phonophobia-free rates at 2 hours, as can be seen from the $X^{2}$ and $I^{2}$ statistics in Figure 2. However, subgroup analysis to investigate the cause of heterogeneity cannot be performed due to insufficiency of available data and trials. Important probable sources of heterogeneous results may include diversity in the proportions of various severities of migraine and differences in the inclusion/exclusion criteria, e.g. exclusion of participants with significant vomiting or who required bed rest in most of the migraine episodes. Results may also vary according to the timing of administration, which might be early or late when the attack is fully developed. ${ }^{10}$

This review holds few usable data and relevant studies to allow various sensitivity analyses. In particular, the effects of missing data on the results were only explored (data not shown). In the comparison of AAC regimen and placebo, per-protocol and intention-to-treat analyses revealed similar favorable results for the AAC regimen in terms of pain-free, headache relief, nausea-free, photophobia-free, phonophobia-free and functional disability reduction rates at 2 hours. Agreement of effect estimates across analyses generates robust results suggesting that AAC may be both efficacious and effective for acute migraine in adults compared to placebo. On the other hand, in the comparison of AAC with active controls, discrepancy in results exists in various analyses. The ITT analyses for pain-free and headache relief rates at 2 hours in the comparison of AAC versus Ibuprofen $400 \mathrm{mg}$ showed discordant results. For the AAC and Sumatriptan $50 \mathrm{mg}$ comparison, the former regimen may be efficacious as showed in the results of headache relief at 2 hours, but ITT analyses showed varying results. Results for relapse rate in the comparison of AAC and Sumatriptan $50 \mathrm{mg}$ were also inconsistent. For AAC versus Sumatriptan $100 \mathrm{mg}$, no outcome measure showed consistent findings across analyses.

Several expert societies have previously recommended the fixed combination of the AAC regimen for the treatment of acute migraine. German, Austrian and Swiss headache societies and the German Society of Neurology, in their guidelines on the self-medication of migraine [and tensiontype headache], AAC regimen was highlighted as the drug of first choice on the basis of comparative studies. ${ }^{15,16}$ In the review published by the journal of the American Family Physician, AAC was regarded as "effective, inexpensive, and available without prescription, and free from vascular contraindications associated with triptans" and should be employed as first-line therapy for migraine attacks based on previously published trials. ${ }^{11,12,18}$ This review, with particular regard to the risk of bias or methodological quality of the included studies, incorporated the effect estimates of other relevant trials, generating more robust results for most of the standard outcomes for acute migraine.

Every step in the review process, which included the screening of records, application of the eligibility criteria for inclusion of studies, data extraction, and data synthesis and analysis, was performed by at least 2 members of the review authors. This design was done to minimize unforeseen error and systematic bias that could transpire if only one author was responsible for each step. Allowing a third author to serve as an arbitrator if disagreements emerge between 2 authors further minimized these possible errors.

The included trials were mainly identified using electronic databases. The systematic search was only done through the most well-known international general healthcare databases and major local electronic databases. In addition, handsearching was done only in relevant, accessible trials and reviews that were identified in the search process. Grey literature was not explored satisfactorily. Moreover, this review only incorporated English-reported trials; however, at the time of systematic search for relevant articles, there were no non-English articles that were found to be eligible for inclusion. Due to 
unavailability of unreported data, this review only pooled the data from the published information of the included trials.

Overall, this review synthesized the available evidences on the effectiveness and tolerability of the AAC regimen for the acute treatment of migraine. Most trials have compared AAC to placebo but there were very few studies dedicated on the head-on comparison of AAC versus active controls. Further research should aim for the determination of the relative effectiveness of this regimen to other standard therapies for migraine. In addition, reports of trials generally do not adhere completely to established guidelines for the selection of outcome measures in the conduct of migraine clinical trials. To improve consistency and to compare straightforwardly the effectiveness of the migraine interventions across trials, important outcomes should be selected from standard and validated guidelines. ${ }^{9}$ Moreover, no trial has measured sustained pain-free rate, an outcome which reflects long term effectiveness of an antimigraine drug; thus, clinical drug trials involving migraineurs should also consider this significant outcome.

\section{Conclusion}

For adults with moderate to severe migraine, a fixed oral dose of Acetaminophen/Aspirin/Caffeine (AAC) at $500 / 500 / 130 \mathrm{mg}$ may be used as first-line therapy for the acute treatment of migraine. This regimen relieves pain, nausea, photophobia, photophobia and functional disability and associated only with mild and infrequent adverse events.

Statement of Authorship

All authors have approved the final version submitted.

\section{Author Disclosure}

All the authors declared no conflicts of interest.

\section{Funding Source}

None.

\section{References}

1. Smitherman TA, Burch R, Sheikh H, Loder E. The prevalence, impact, and treatment of migraine and severe headaches in the United States: a review of statistics from national surveillance studies. Headache. 2013; 53(3):427-36.
2. Lipton RB, Stewart WF, Diamond S, Diamond ML, Reed M. Prevalence and burden of migraine in the United States: data from the American Migraine Study II. Headache. 2001; 41(7):646-57.

3. Stewart WF, Roy J, Lipton RB. Migraine prevalence, socioeconomic status, and social causation. Neurology. 2013; 81(11):948-55.

4. Anneken K, Evers S, Husstedt IW. Efficacy of fixed combinations of acetylsalicyclic acid, acetaminophen and caffeine in the treatment of idiopathic headache: a review. Eur J Neurol. 2010; 17(4):534-e25.

5. Straube A, Aicher B, Fiebich BL, Haag G. Combined analgesics in (headache) pain therapy: shotgun approach or precise multi-target therapeutics? BMC Neurol. 2011; 11:43.

6. Higgins J, Green S. Cochrane handbook for systematic reviews of interventions version 5.1.0 [Online]. The Cochrane Collaboration. 2011 [cited 2014 Jan]. Available from www.cochrane-handbook.org.

7. Liberati A, Altman DG, Tetzlaff J, et al. The PRISMA statement for reporting systematic reviews and meta-analyses of studies that evaluate health care interventions: explanation and elaboration. BMJ. 2009; 339:b2700.

8. International Headache Society. The International Classification of Headache Disorders, $2^{\text {nd }}$ Edition. Cephalalgia. 2004; 24 Suppl 1:9-160.

9. Tfelt-Hansen P, Pascual J, Ramadan N, et al. Guidelines for controlled trials of drugs in migraine: a guide for investigators (third edition). A guide for investigators. Cephalalgia. 2012; 32(1):6-38.

10. Review Manager (RevMan). Version 5.2. 2012. Copenhagen: The Nordic Cochrane Centre, The Cochrane Collaboration.

11. Goldstein J, Silberstein SD, Saper JR, et al. Acetaminophen, aspirin, and caffeine versus sumatriptan succinate in the early treatment of migraine: results from the ASSET trial. Headache. 2005; 45(8):973-82.

12. Goldstein J, Silberstein SD, Saper JR, Ryan RE Jr, Lipton RB. Acetaminophen, aspirin, and caffeine in combination versus ibuprofen for acute migraine: results from a multicenter, double-blind, randomized, parallel-group, single-dose, placebo-controlled study. Headache. 2006; 46(3):444-53.

13. Lipton RB, Stewart WF, Ryan RE Jr, Saper J, Silberstein S, Sheftell F. Efficacy and safety of acetaminophen, aspirin, and caffeine in alleviating migraine headache pain: three double-blind, randomized, placebocontrolled trials. Arch Neurol. 1998; 55(2):210-7.

14. Clinicaltrials.gov. Efficacy and Safety of Acetaminophen, Aspirin and Caffeine with Sumatriptan in the Acute Treatment of Migraine (Phase 4) [NCT01248468] [Online]. 2012 (cited 2014 Jan]. Available from http://clinicaltrials.gov/show/NCT01248468.

15. Haag G, Diener HC, May A, et al. Self-medication of migraine and tension-type headache: summary of the evidence-based recommendations of the Deutsche Migräne und Kopfschmerzgesellschaft (DMKG), the Deutsche Gesellschaft für Neurologie (DGN), the Österreichische Kopfschmerzgesellschaft (ÖKSG) and the Schweizerische Kopfwehgesellschaft (SKG). J Headache Pain. 2011; 12(2):201-17.

16. Diener HC, Pfaffenrath V, Pageler L, Peil H, Aicher B. The fixed combination of acetylsalicylic acid, paracetamol and caffeine is more effective than single substances and dual combination for the treatment of headache: a multicentre, randomized, double-blind, single-dose, placebo-controlled parallel group study. Cephalalgia. 2005; 25(10):776-87.

17. Diener HC, Dowson AJ, Ferrari M, Nappi G, Tfelt-Hansen P. Unbalanced randomization influences placebo response: scientific versus ethical issues around the use of placebo in migraine trials. Cephalalgia 1999; 19(8):699-700.

18. Gilmore B, Michael M. Treatment of acute migraine headache. Am Fam Physician. 2011; 83(3):271-80. 